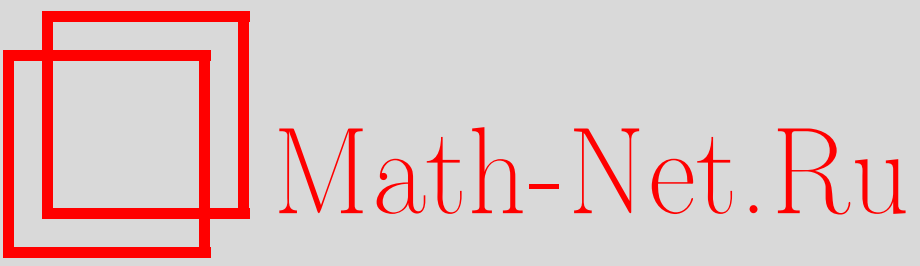

В. В. Власов, О некоторых спектральных вопросах, возникающих в теории дифференциально-разностных уравнений, УМH, 1998, том 53, выпуск 4, $217-$ 218

DOI: https://doi.org/10.4213/rm65

Использование Общероссийского математического портала Math-Net.Ru подразумевает, что вы прочитали и согласны с пользовательским соглашением http://www . mathnet.ru/rus/agreement

Параметры загрузки:

IP: 34.229 .108 .108

26 апреля 2023 г., 09:36:52 


\title{
О НЕКОТОРЫХ СПЕКТРАЛЬНЫХ ВОПРОСАХ, ВОЗНИКАЮЩИХ В ТЕОРИИ ДИФФЕРЕНЦИАЛЬНО-РАЗНОСТНЫХ УРАВНЕНИЙ
}

\author{
В.В. ВлАСОВ
}

В предлагаемой заметке рассмотрены некоторые спектральные вопросы, естественно возникающие в теории дифференциально-разностных уравнений, включающие в себя изучение полноты и базисности Рисса системы экспоненциальных (элементарных) решений, а также установлены оценки (в определенном смысле неулучшаемые) сильных решений упомянутых уравнений.

Рассмотрим следующую начальную задачу

$$
\begin{gathered}
\sum_{j=0}^{n}\left(B_{j} u\left(t-h_{j}\right)+D_{j} \frac{d u}{d t}\left(t-h_{j}\right)\right)+\int_{0}^{h} K(s) u(t-s) d s=0, \quad t>0, \\
u(t)=y(t), \quad t \in[-h, 0), \quad u(+0)=\varphi_{0}=y(-0) .
\end{gathered}
$$

Здесь $B_{j}, D_{j}(j=0,1, \ldots, n)$ - матрицы размера $m \times m$ с постоянными комплексными элементами, числа $h_{j}$ таковы, что $0=h_{0}<h_{1}<\cdots<h_{n}=h$, элементы $K_{i j}(s)(i, j=1,2, \ldots, m)$ матрицы $K(s)$ принадлежат пространству $L_{1}(0, h)$.

Обозначим через $l(\lambda)$ характеристический квазимногочлен (см. [1], [4]) уравнения (1), через $\lambda_{q}$ - нули функции $l(\lambda)$, упорядоченные в порядке возрастания модулей, через $\nu_{q}-$ их кратности, через $\Lambda$ - множество всех нулей функции $l(\lambda)$, через $y_{q, j, s}(t)\left(j=1,2, \ldots, p_{q}, s=0,1, \ldots, r_{p q}\right)$ - экспоненциальные решения (см. [3], [4]) уравнения (1).

Обозначим через $W_{2, \gamma}^{p}\left((a, b), \mathbb{C}^{m}\right),(-\infty<a<b \leqslant+\infty), p=1,2, \ldots$, весовые пространства Соболева вектор-функций со значениями в $\mathbb{C}^{m}$, снабженные нормами

$$
\|u\|_{W_{2, \gamma}^{p}(a, b)} \equiv\left(\int_{a}^{b} \exp (-2 \gamma t)\left(\sum_{j=0}^{p}\left\|u^{(j)}(t)\right\|_{\mathbb{C}^{m}}^{2}\right) d t\right)^{1 / 2}, \quad \gamma \in \mathbb{R} .
$$

Здесь и в дальнейшем полагаем $W_{2,0}^{p}=W_{2}^{p}, u^{(p)}(t)=\frac{d^{p}}{d t^{p}} u(t), p=1,2 \ldots$.

ОПРЕДЕЛЕНИЕ. Функцию $u(t)$, принадлежащую пространству $W_{2, \gamma}^{1}\left((-h,+\infty), \mathbb{C}^{m}\right)$ при некотором $\gamma \in \mathbb{R}_{+}$, назовем сильным решением задачи $(1),(2)$, если $u(t)$ удовлетворяет уравнению (1) при почти всех $t \in \mathbb{R}_{+}=(0,+\infty)$, а также условию (2).

Лемма 1. Пусть $\operatorname{det} D_{0} \neq 0$. Тогда $\varkappa_{+}=\sup _{\lambda_{q}} \operatorname{Re} \lambda_{q}<\infty$ и для любого $\gamma \geqslant \varkappa_{+}+\varepsilon$ $(\varepsilon>0)$ задача $(1),(2)$ однозначно разрешима в пространстве $W_{2, \gamma}^{1}\left((-h,+\infty), \mathbb{C}^{m}\right)$ для произвольной вектор-функции $y(t) \in W_{2}^{1}\left((-h, 0), \mathbb{C}^{m}\right)$, причем для ее решения $и(t)$ справедливо неравенство

$$
\|u\|_{W_{2, \gamma}^{1}(-h,+\infty)} \leqslant d_{\varepsilon}\|y\|_{W_{2}^{1}(-h, 0)}
$$

c постоянной $d_{\varepsilon}$, не зависящей от функции $y(t)$.

Принимая во внимание лемму 1 , введем аналогично [2] полугруппу $\mathbb{V}_{t}$ ограниченных операторов, действующих в пространстве $W_{2}^{1}\left((-h, 0), \mathbb{C}^{m}\right)$ согласно правилу $\left(\mathbb{V}_{t} y\right)(s)=u(t+s)$, $t \geqslant 0, s \in[-h, 0]$, где $u(\cdot)$ - решение задачи $(1),(2)$, отвечающее начальной функции $y(s)$.

Лемма 2. Пусть $\operatorname{det} D_{0} \neq 0$. Тогда семейство операторов $\mathbb{V}_{t}(t \geqslant 0)$ образует $C^{0}$ - полугруппу в пространстве $W_{2}^{1}\left((-h, 0), \mathbb{C}^{m}\right)$ с генератором $\mathbb{D}$, имеющим область определения

$\operatorname{Dom}(\mathbb{D})=\left\{\varphi \in W_{2}^{2}\left((-h, 0), \mathbb{C}^{m}\right), \sum_{j=0}^{n}\left(B_{j} \varphi\left(-h_{j}\right)+D_{j} \varphi^{(1)}\left(-h_{j}\right)\right)+\int_{0}^{h} K(s) \varphi(-s) d s=0\right\}$

и действующим по правилу $(\mathbb{D} \varphi)(s)=\varphi^{(1)}(s), s \in(-h, 0)$, причем решения $y_{q, j, s}(t)$ являются его корневыми векторами, отвечающими собственным значениям $\lambda_{q}$.

Работа выполнена при финансовой поддержке Российского фонда фундаментальных исследований (гранты №№ 96-01-00333, 96-15-96091). 
Teоpema 1. Пусть $\operatorname{det} D_{0} \neq 0, \operatorname{det} D_{n} \neq 0$. Tогдa:

1. резольвента $R(\lambda, \mathbb{D})$ оператора $\mathbb{D}$ является конечномероморфной, а система корневых векторов $\left\{y_{q, j, s}(t)\right\}$ оператора $\mathbb{D}$ полна и минимальна в пространстве $W_{2}^{1}\left((-h, 0), \mathbb{C}^{m}\right)$

2. конечнь величинь $\varkappa_{-}=\inf _{\lambda_{q} \in \Lambda} \operatorname{Re} \lambda_{q}, N=\max _{\lambda_{q} \in \Lambda} \nu_{q}$;

3. существуют положительные постоянные $\delta$ и $\Delta$, а также последовательность отрезков $r_{n}=\left(t+i c_{n}\right), t \in\left[\varkappa_{-}-\beta, \varkappa_{+}+\beta\right], \beta>0,0<\delta \leqslant c_{n+1}-c_{n} \leqslant \Delta<+\infty, n \in \mathbb{Z}$, принадлежащих области голоморфности резольвенты $R(\lambda, \mathbb{D})$, таких, что количество $\mu_{n}$ нулей $\lambda_{q}$ (с учетом кратности), лежащих в прямоугольниках $\Gamma_{n}$, сторонами которьх являются отрезки $r_{n}$ и $r_{n+1}$, а также отрезки $\left(\varkappa_{-}-\beta\right)+i \tau,\left(\varkappa_{+}+\beta\right)+i \tau$, $\tau \in\left[c_{n}, c_{n+1}\right]$, равномерно ограничено по $n$ :

$$
\max _{n \in \mathbb{Z}} \mu_{n} \leqslant M
$$

4. система подпространств $\mathscr{W}_{n}=\mathscr{P}_{n} W_{2}^{1}\left((-h, 0), \mathbb{C}^{m}\right), n \in \mathbb{Z}$, где $\mathscr{P}_{n}-$ риссовские спектральные проекторь оператора $\mathbb{D}$, отвечающие контурам $\partial \Gamma_{n}$ (границам $\left.\Gamma_{n}\right)$, образует базис Рисса из подпространств пространства $W_{2}^{1}\left((-h, 0), \mathbb{C}^{m}\right)$.

СледСтвиЕ 1. Пусть $\operatorname{det} D_{0} \neq 0$, det $D_{n} \neq 0$, причем $\inf _{\lambda_{p} \neq \lambda_{q}}\left|\lambda_{p}-\lambda_{q}\right|>0$.

Тогда система подпространств $V_{\lambda_{q}}=\operatorname{Span}\left\{y_{q, j, s}(t), j=\overline{1, p_{q}} ; s=\overline{0, r_{p q}}\right\}$ образует базис Рисса из подпространств пространства $W_{2}^{1}\left((-h, 0), \mathbb{C}^{m}\right)$.

Теорема 2. Пусть $\operatorname{det} D_{0} \neq 0, \operatorname{det} D_{n} \neq 0$. Тогда для любого сильного решения $u(t)$ задачи (1), (2) выполнено неравенство

(6) $\|u(t+\cdot)\|_{W_{2}^{1}(-h, 0)} \equiv\left\|\left(\mathbb{V}_{t} y\right)(s)\right\|_{W_{2}^{1}(-h, 0)} \leqslant d(t+1)^{M-1} \exp \left(\varkappa_{+} t\right)\|y\|_{W_{2}^{1}(-h, 0)}, \quad t \geqslant 0$,

с постоянной $d$, не зависящей от начальной функции $y(s)$, и постоянной $M$, определяемой в пункте 3 теоремы 1.

СлЕДСТВИЕ 2. Пусть выполнены условия следствия 1. Тогда для любого сильного решения $и(t)$ задачи (1), (2) справедливо неравенство вида (6), в котором постоянная $M$ заменяется на постоянную $N=\max _{\lambda_{q} \in \Lambda} \nu_{q}$.

Теорема 3. Пусть det $D_{0} \neq 0, \inf _{\lambda_{p} \neq \lambda_{q}}\left|\lambda_{p}-\lambda_{q}\right|>0, K(s) \equiv 0$. Тогда справедливо утверждение теоремы 2 , причем (6) справедливо с постоянной $M=N=\max _{\lambda_{q} \in \Lambda} \nu_{q}$.

ЗАмечАниЕ 1 . Оценка, аналогичная $(6)$, для которой величина $\varkappa_{+}$заменяется на $\left(\varkappa_{+}+\varepsilon\right)$, $\varepsilon>0$, давно и хорошо известна (см. [1], [2]). В этой связи возникал [2] вопрос: можно ли положить $\varepsilon=0$ ? Теоремы 2 и 3 дают в определенном смысле утвердительный ответ на данньй вопрос.

В заключение отметим, что теоремы 1-3 и их следствия существенно усиливают и развивают соответствующие резултаты из [3], [4]. При ином понимании решений базисность и полнота экспоненциальных решений в пространстве $\mathbb{C}^{m} \oplus L_{2}\left((-h, 0), \mathbb{C}^{m}\right)$ рассматривалась в [5].

Автор благодарит А. Г. Костюченко за внимание к работе.

\section{СПИСОК ЛИТЕРАТУРЫ}

[1] Беллман Р., Кук К. Дифференциально-разностные уравнения. М.: Мир, 1967. [2] Хейл Дж. Теория функционально-дифференциальных уравнений. М.: Мир, 1984. [3] Власов В. В. // Известия вузов. Математика. 1996. №1. С. 22-35. [4] Власов В.В.// УМH. 1996. T. 51. № 1. C. 143-144. [5] Verduyn Lunel S., Yakubovich D. V. // Integr. Eq.\& Oper. Theory. 1997. V. 27. P. 347-378.

Московский физико-технический институт

Принято редколлегией 03.06.1998 